PROCEEDINGS OF THE

AMERICAN MATHEMATICAL SOCIETY

Volume 132, Number 9, Pages 2505-2509

S 0002-9939(04)07469-6

Article electronically published on April 8, 2004

\title{
ON RINGS WITH SMALL HILBERT-KUNZ MULTIPLICITY
}

\author{
MANUEL BLICKLE AND FLORIAN ENESCU
}

(Communicated by Bernd Ulrich)

\begin{abstract}
A result of Watanabe and Yoshida says that an unmixed local ring of positive characteristic is regular if and only if its Hilbert-Kunz multiplicity is one. We show that, for fixed $p$ and $d$, there exists a number $\epsilon(d, p)>0$ such that for any nonregular unmixed ring $R$ its Hilbert-Kunz multiplicity is at least $1+\epsilon(d, p)$. We also show that local rings with sufficiently small Hilbert-Kunz multiplicity are Cohen-Macaulay and $F$-rational.
\end{abstract}

\section{INTRODUCTION}

By a result of Watanabe and Yoshida [7, an unmixed local ring $R$ of characteristic $p>0$ is regular if and only if the Hilbert-Kunz multiplicity,

$$
e_{H K}(R)=\lim _{e \rightarrow \infty} \frac{\lambda\left(R / \mathfrak{m}^{\left[p^{e}\right]}\right)}{p^{d e}},
$$

is equal to one. A short proof of this was given by Huneke and Yao in 6]. The Hilbert-Kunz multiplicity has proven to be difficult to compute, and even basic questions, such as whether it is rational, defied a solution despite intense effort. The above result raises the following, also very basic, question: What is the smallest possible Hilbert-Kunz multiplicity of a nonregular unmixed ring, say of fixed dimension $d$ and characteristic $p$ ? That is, we ask to determine the number

$$
\epsilon_{H K}(d, p)=\inf \left\{e_{H K}(R)-1: R \text { nonregular, unmixed, } \operatorname{dim} R=d, \operatorname{char} R=p\right\} .
$$

In this paper we start a first investigation into this question by showing that, at least, $\epsilon_{H K}(d, p)$ is always strictly positive, i.e., the Hilbert-Kunz multiplicity of a nonregular ring of fixed dimension and characteristic cannot be arbitrarily close to one. The bound we obtain is by no means optimal as the few cases, where $\epsilon_{H K}(d, p)$ is known, show.

In the course of our investigation we obtain a result of a similar flavor. We show that a small Hilbert-Kunz multiplicity, precisely $e_{H K}(R) \leq 1+1 / d$ !, implies Cohen-Macaulayness and $F$-rationality (see Proposition 2.5). This sheds some light into the difficult task of determining the subtle manner in which the Hilbert-Kunz multiplicity encodes information about the singularity of $R$.

Received by the editors October 31, 2002.

2000 Mathematics Subject Classification. Primary 13A35.

Key words and phrases. Regular rings, Hilbert-Kunz multiplicity, F-rational rings.

(C) 2004 by the authors 


\section{LOWER BOUndS ON THE HilBerT-KUnZ MULTIPLICITY}

We fix the notation of a $d$-dimensional local ring $(R, \mathfrak{m}, k)$ with maximal ideal $\mathfrak{m}$ and residue field $k$ of characteristic $p>0$. For every positive integer $e$, let $q=p^{e}$. If $I$ is an ideal of $R$, then $I^{[q]}=\left(i^{q}: i \in I\right)$.

Definition 2.1. Let $I$ be an $\mathfrak{m}$-primary ideal in $R$. The Hilbert-Samuel multiplicity of $I$ with respect to $R$ is defined by

$$
\lim _{n \rightarrow \infty} \frac{d ! \lambda\left(R / I^{n}\right)}{n^{d}} .
$$

This limit exists and is denoted by $e(I, R)$, or, simply, by $e(I)$. The Hilbert-Kunz multiplicity of $I$ with respect to $R$ is defined by

$$
\lim _{n \rightarrow \infty} \frac{\lambda\left(R / I^{[q]}\right)}{q^{d}}
$$

This limit exists and is denoted by $e_{H K}(I, R)$, or, simply, by $e_{H K}(I)$. The number $e(\mathfrak{m}, R)$ is called the Hilbert-Samuel multiplicity of $R$ and is usually denoted by $e(R)$.

For a collection of basic results regarding these notions, we refer the reader to [5], or [7]. We list here only a few well-known facts that will be needed later.

One has $\lambda\left(R / \mathfrak{m}^{[q]}\right) \geq q^{d}$, and the equality for one particular $q$ implies that $R$ is regular and hence that $\lambda\left(R / \mathfrak{m}^{[q]}\right)=q^{d}$ for all $q$. In general, $\max (1, e(I) / d !) \leq$ $e_{H K}(I) \leq e(I)$, for every $\mathfrak{m}$-primary ideal of $R$. If $I$ is generated by a system of parameters, then $e(I)=e_{H K}(I)$. In [4] Hanes recently showed that, in fact, $e(I) / d !<e_{H K}(I)$, for every $\mathfrak{m}$-primary ideal $I$, answering affirmatively a question raised by Watanabe and Yoshida (Question 2.9 in [7]).

In our paper, we look at the class of unmixed rings. We say that $R$ is unmixed if all the associated primes have codimension equal to the dimension of $R$. We restrict ourselves to this case, because there are examples of nonregular rings that are not unmixed with $e_{H K}=1$. We will also assume that $d \geq 2$, since in the onedimensional case the Hilbert-Kunz multiplicity is at least 2 for nonregular rings.

The following lemma, although easy to prove, turns out to be a very useful tool in many instances (see 6], Lemma 2.1, or 7], Lemma 4.2).

Lemma 2.2. Let $(R, \mathfrak{m}, k)$ be of characteristic $p>0$.

(1) $e_{H K}(I) \leq \lambda\left(R / I^{*}\right) \cdot e_{H K}(R)$.

(2) For $I$ an $\mathfrak{m}$-primary ideal of $R$, denote $f_{I}=\lambda(R / I) e_{H K}(R)-e_{H K}(I)$. Then, for every pair of ideals $J \subset I$ one has $f_{I} \leq f_{J}$.

The following theorem was conjectured by Watanabe and Yoshida and is the key tool in our investigation.

Theorem 2.3 (Goto-Nakamura, [3]). Let $(R, \mathfrak{m})$ be a homomorphic image of a Cohen-Macaulay local ring of characteristic $p>0$.

(1) Assume that $R$ is equidimensional. Then $e(I) \geq \lambda\left(R / I^{*}\right)$ for every parameter ideal $I$.

(2) Assume that $R$ is unmixed. If $e(I)=\lambda\left(R / I^{*}\right)$ for some parameter ideal $I$, then $R$ is a Cohen-Macaulay F-rational local ring.

Remark 2.4. An alternate proof of this theorem has also been given by Ciupercă and Enescu, [2] (Theorem 1.1 and Remark 1.10), where (2) is obtained under some other mild conditions on $R$. 
First we present a result which shows that rings with sufficiently small HilbertKunz multiplicity are Cohen-Macaulay and $F$-rational.

Proposition 2.5. Let $R$ be an unmixed ring that is a homomorphic image of a Cohen-Macaulay local ring of characteristic $p>0$. If $e_{H K}(R) \leq 1+$ $\max \{1 / d !, 1 / e(R)\}$, then $R$ is Cohen-Macaulay and F-rational.

Proof. Since the associated primes of $R$ and $R[x]_{\mathfrak{m}}$ are in bijection, $R$ is unmixed if and only if $R[x]_{\mathfrak{m}}$ is unmixed. The same holds for the multiplicities, CohenMacaulayness and $F$-rationality. Thus we can assume that the residue field of $R$ is infinite. Hence, we can choose a minimal reduction ideal $I$ for $\mathfrak{m}$ (i.e., $e(I)=$ $e(\mathfrak{m})=e(R))$ such that $I$ is generated by a system of parameters.

Let us assume that $R$ is not Cohen-Macaulay and $F$-rational. We show that this implies that $e_{H K}(R)>1+1 / e(R)$. By Lemma 2.2 we have that $e(I) \leq$ $\lambda\left(R / I^{*}\right) e_{H K}(R)$ and substituting $\lambda\left(R / I^{*}\right) \leq e(R)-1$ (Theorem [2.3) we get $e(I) \leq$ $(e(I)-1) e_{H K}(R)$. Thus,

$$
e_{H K}(R) \geq 1+\frac{1}{e(R)-1}>1+\frac{1}{e(R)} .
$$

To see that $e_{H K}(R)>1+1 / d$ !, it is now enough to consider the case $d ! \leq e(R)-1$. Then we have

$$
e_{H K}(R)>\frac{e(R)}{d !} \geq \frac{d !+1}{d !}=1+\frac{1}{d !},
$$

finishing the argument.

Remark 2.6. Watanabe and Yoshida ([7, [8]) have shown that, in dimension 2, under the assumption of Cohen-Macaulayness, the minimal Hilbert-Kunz multiplicity is at least 3/2. Our Proposition 2.5 shows that, in fact, this assumption can be dropped in the case of unmixed rings that are a homomorphic image of a Cohen-Macaulay ring.

Now we can address the existence of a lower bound for the Hilbert-Kunz multiplicity of nonregular rings.

Theorem 2.7. Let $(R, \mathfrak{m}, k)$ be an unmixed local ring that is the homomorphic image of a Cohen-Macaulay ring of characteristic $p>0$ and dimension $d$. If $R$ is not regular, then $e_{H K}(R)>1+\max \left\{1 /\left(p^{d} d !\right), 1 /\left(p^{d} e(R)\right)\right\}$.

Proof. As before we can assume that the residue field is infinite and let $I$ be a minimal reduction for $\mathfrak{m}$. Proposition 2.5 shows that it is enough to consider the case when $R$ is Cohen-Macaulay and $F$-rational.

By Lemma 2.2 we have $f_{\mathfrak{m}[p]} \leq f_{I^{[p]}}$. Substituting into this inequality the identities $e_{H K}\left(I^{[p]}\right)=e_{H K}(I) p^{d}, e\left(I^{[p]}\right)=\lambda\left(R / I^{[p]}\right)$ and $e\left(I^{[p]}\right)=e_{H K}\left(I^{[p]}\right)$ we get

$$
e_{H K}(R) \cdot\left(\lambda\left(R / \mathfrak{m}^{[p]}\right)-p^{d}\right) \leq e\left(I^{[p]}\right) \cdot\left(e_{H K}-1\right)=p^{d} e(R) \cdot\left(e_{H K}-1\right) .
$$

If $R$ is not regular, then $\left(\lambda\left(R / \mathfrak{m}^{[p]}\right)-p^{d}\right) \geq 1$ and we obtain from (1), by solving for $e_{H K}(R)$, that

$$
e_{H K}(R) \geq 1+\frac{1}{p^{d} e(R)-1}>1+\frac{1}{p^{d} e(R)} .
$$

Using the inequality $e(R)<d ! e_{H K}(R)$ in the right side of (1) we obtain similarly that

$$
e_{H K}(R)>1+\frac{1}{p^{d} d !} \text {. }
$$


Remark 2.8. Note that this given bound by no means improves the trivial bound for rings $R$ with $e(R)>d$ ! given by

$$
e_{H K}(R)>e(R) / d ! \geq(d !+1) / d !=1+1 / d ! .
$$

The the more interesting case is that of comparatively small multiplicity $e(R)$ in which our bound gives new results.

Our results are obtained by manipulating inequalities involving $e_{H K}(R), e(R)$ and the colength of various m-primary ideals. Improving these inequalitites will result in improving the bounds on the Hilbert-Kunz multiplicity. In what follows, we present a situation like this. Hanes has recently proved the inequality

$$
e_{H K} \geq e(R) \cdot \frac{\nu}{\left(\nu^{1 /(d-1)}-1\right)^{d-1}}
$$

where $\nu$ is the minimal number of generators for $\mathfrak{m}$. But $e(R) \geq \nu-d+1$; so, in fact,

$$
e_{H K} \geq \frac{\nu-d+1}{d !} \cdot \frac{\nu}{\left(\nu^{1 /(d-1)}-1\right)^{d-1}} .
$$

Using this inequality, Hanes obtains lower bounds for the Hilbert-Kunz multiplicity of nonregular rings. He notices that the function

$$
f(\nu)=\frac{\nu-d+1}{d !} \cdot \frac{\nu}{\left(\nu^{1 /(d-1)}-1\right)^{d-1}}
$$

is increasing. So $e_{H K} \geq f(d+1)$ (since $\nu \geq d+1$ ). For $d \leq 4, f(d+1)$ is greater than 1 and lower bounds for the Hilbert-Kunz multiplicity are obtained.

Let us show how we can use this circle of ideas to improve our bounds. First note that if $R$ is not Cohen-Macaulay, then $e_{H K} \geq f(d+2)(\nu=d+2$ implies that $R$ is complete intersection, hence Cohen-Macaulay).

Proposition 2.9. Let $(R, \mathfrak{m}, k)$ be an unmixed local ring of dimension $d$. If $e_{H K}(R) \leq 1+f(d+2)$, then $R$ is Cohen-Macaulay and F-rational.

Proof. Assume that $e_{H K}(R) \leq 1+f(d+2)$. As previously shown, if $R$ is not Cohen-Macaulay and $F$-rational, then $e(R) \geq 1 / f(d+2)+1$. Hence, $e_{H K} \geq$ $e(R) f(\nu) /(\nu-d+1) \geq(1+f(d+2)) /(\nu-d+1)$.

\section{Proposed Problems}

A shortcoming of the bound of Theorem 2.7] is its dependence upon the characteristic. In fact, we suspect that the number

$$
\epsilon_{H K}(d)=\inf \left\{\epsilon_{H K}(d, p): p>0\right\}
$$

is strictly bigger than 0 . In the one-dimensional case this is trivial, $\epsilon_{H K}(1)=1$. Work of Watanabe and Yoshida [7, together with our Remark 2.6, shows that $\epsilon_{H K}(2)=1 / 2$. Furthermore, Watanabe and Yoshida give a classification of all twodimensional Cohen-Macaulay rings with Hilbert-Kunz multiplicity less than 2. Up to dimension 4, Hanes's results, [4, also give lower bounds for $\epsilon_{H K}(d, p)$ that are independent of $p$ (he can show that $\epsilon_{H K}(3) \geq 1 / 3$, and that $\epsilon_{H K}(4, p) \geq 0.16$ ). In private communications, Watanabe mentioned that he and Yoshida can also prove that $\epsilon_{H K}(3)=1 / 3$ in dimension 3. In dimensions 2 and 3, Buchweitz and Chen have obtained the minimal Hilbert-Kunz multiplicity of homogeneous hypersurfaces 
of fixed degree (see [1]). It should be noted that none of these techniques is known to produce bounds in the general case.

Another question that comes to mind is whether $\epsilon_{H K}(d, p)$ is attained. If this is the case, what is the significance of such rings with minimal Hilbert-Kunz multiplicity? Are they unique up to isomorphism? Again, the work of Watanabe and Yoshida gives an answer in dimension two only, and all remaining dimensions remain completely open.

In view of Proposition 2.5 we can shift attention away from nonregular rings to other classes of singularities, and ask for the minimal Hilbert-Kunz multiplicity of a $d$-dimensional ring with a prescribed singularity. The intuition behind this is that bad singularities should force a high Hilbert-Kunz multiplicity. Our Proposition 2.5 is a result of this type.

\section{ACKNOWLEDGMENTS}

The second author would like to thank Craig Huneke and Kei-ichi Watanabe for useful discussions and Douglas Hanes for providing him with a preprint of his work and interesting correspondence. The final version of this paper was written while the authors were visiting the Mathematical Research Sciences Institute at Berkeley, California, and they would like to thank this institution for a great work environment and financial support.

\section{REFERENCES}

[1] R.-O. Buchweitz and Q. Chen, Hilbert-Kunz Functions of Cubic Curves and Surfaces, J. Algebra 197, 246-267 (1997). MR 98i:13025

[2] C. Ciupercă and F. Enescu, An inequality involving tight closure and parameter ideals, preprint, 2002.

[3] S. Goto and Y. Nakamura, Multiplicity and tight closures of parameter ideals, J. Algebra 244, 302-311 (2001). MR 2002h:13008

[4] D. Hanes, Notes on the Hilbert-Kunz function, J. Algebra 265, 619-630 (2003).

[5] C. Huneke, Tight closure and its applications, with an appendix by Melvin Hochster, CBMS Regional Conference Series in Mathematics, 88, American Mathematical Society, Providence, RI, 1996. MR 96m:13001

[6] C. Huneke and Y. Yao, Unmixed local rings with minimal Hilbert-Kunz multiplicity are regular, Proc. Amer. Math. Soc. 130, 661-665 (2002). MR 2002h:13026

[7] K.-i. Watanabe and K. Yoshida, Hilbert-Kunz Multiplicity and an Inequality between Multiplicity and Colength, J. Algebra 230, 295-317 (2000). MR 2001h:13032

[8] K.-i. Watanabe and K. Yoshida, Hilbert-Kunz multiplicity of two-dimensional local rings, Nagoya Math. J. 162, 87-110 (2001). MR 2002e:13040

FB6 Mathematik, Universität Essen, 45117 Essen, Germany

E-mail address: manuel.blickle@uni-essen.de

Department of Mathematics, University of Utah, Salt lake City, Utah 84112; Institute of Mathematics of the Romanian Academy, Bucharest, Romania

E-mail address: enescu@math.utah.edu 